\title{
Control of high power microwave radiation by electromagnetic band gap structures
}

\author{
Leanid Simonchik ${ }^{1, *}$, Vladislav Babitski ${ }^{1}$, Thierry Callegari ${ }^{2}$, Jerome Sokoloff $^{2}$ and Maxim Usachonak $^{1}$ \\ ${ }^{1}$ Institute of Physics of NAS of Belarus, 220072 Minsk, Belarus \\ ${ }^{2}$ LAPLACE, UPS-CNRS, 31062 Toulouse, France
}

\begin{abstract}
A plasma control of powerful microwave propagation through the $1 \mathrm{D}$ and 2D EBG structures is investigated. Pulsed discharges in argon (or helium) at atmospheric pressure are applied as the plasma inhomogeneities. Temporal behavior of electron concentration in discharge is determined. The timedependent transmission spectra of 1D EBG structure formed solely by plasma in the X-waveguide are measured. The amplitudes of short $(200 \mathrm{~ns})$ powerful $(50 \mathrm{~kW})$ microwave pulses at frequency of $9.15 \mathrm{GHz}$ are strongly suppressed (more than $40 \mathrm{~dB}$ ) when they fall in the time interval of plasma structure existing. The propagation of these powerful microwave pulses through the triangle metallic 2D EBG structure with the plasma control elements is investigated too: when plasma acts as a compensator of defect in the front row of EBG structure the transmission in direction of $45^{\circ}$ quickly ceases (during a few tenth of nanoseconds), or one quickly arises, when plasma is as an additional defect.
\end{abstract}

\section{Introduction}

During the last decade, there has been an increasing interest in the microwave (MW) reconfigurable devices the transmitted properties of whose are changed by variation of external electric or magnetic fields or other factors. To create electrically tunable devices based, for instance, on the use of electromagnetic band gap (EBG) structures, their some passive elements must be replaced by active ones. However some critical problems for high-speed, large scale or complex systems which use computers or automation servers are occured at that. It can be a significant time delay or an operation at low microwave power or an extra development cost. At the same time, the discharge plasma has a great potential for the application in microwave devices in the function of control elements due to its possibility for variations in size, geometry and density by changing the discharge current [1-3]. In this work, we demonstrate the ability to control the propagation of MW radiation at high (about $50 \mathrm{~kW}$ ) power by one- and two-dimension (1D and 2D) EBG structures using the pulse discharges in argon or helium at atmospheric pressure.

\section{Control by 1D EBG structure}

\subsection{Experimental arrangement}

We developed an 1D EBG structure in a X-band waveguide (WR90) using three pulse discharges, which are arranged along waveguide with the period of $30 \mathrm{~mm}$ (Fig. 1). This period corresponds to the wavelength $\Lambda_{0}$ in

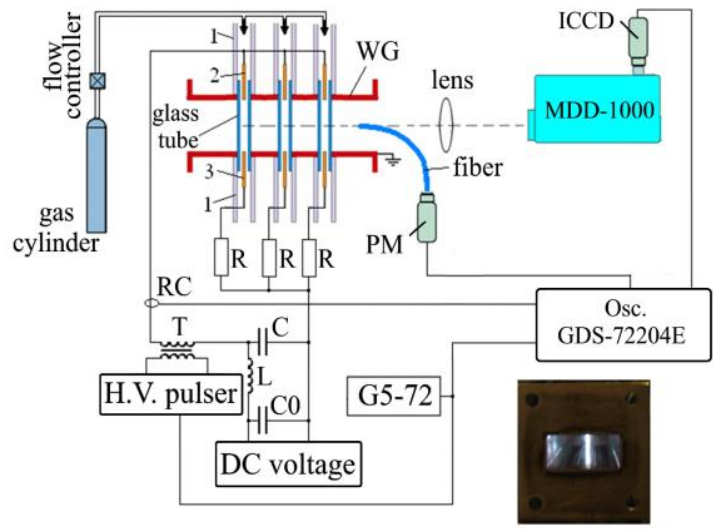

Fig. 1. Schematic of experimental setup. 1 - silicon tubes, 2 and 3 - copper electrodes, WG - waveguide, $\mathrm{R}$ - ballast resistors, $\mathrm{T}-$ transformer, $\mathrm{L}-$ throttle, $\mathrm{C}$ and $\mathrm{C} 0$ - capacitors, $\mathrm{RC}$ - Rogovskii coil. The image of discharge in waveguide is at the right inferior angle.

waveguide for frequency about $11.5 \mathrm{GHz}$ determined by the equation:

$$
\Lambda_{0}=\lambda_{0}\left(1-\left(\lambda_{0} / 2 a\right)^{2}\right)^{-1 / 2},
$$

where $a$ is the wide wall dimension of waveguide and $\lambda_{0}$ is the wavelength in air.

The discharges are ignited in quartz tubes with inner diameters of $1.6 \mathrm{~mm}$, wherethrough argon or helium flows $(\sim 0.5 \mathrm{slm})$ at atmospheric pressure are provided. The electrodes $(2,3)$ with diameters about $1 \mathrm{~mm}$ are fixed inside tubes. In the experiments, the electrode gap is set at $11-12 \mathrm{~mm}$. 
The ignition of discharges is carried out by a short pulse power supply (H.V. pulser) through a pulse transformer T and capacitor C. Simultaneously, capacitor C0 $(4 \mu \mathrm{F})$ charged from a DC voltage source (Fig. 1) begins to discharge through the choke L, the secondary winding of the pulse transformer $\mathrm{T}$ and the electrically broken down gaps in three tubes. The image of discharge in waveguide produced by Canon PowerShot A530 camera is shown in Fig. 1 (in the right inferior angle).

\subsection{Results and discussion}

Typical time evolution of single discharge current is shown in Fig. 2 (solid curve). It is shown that the discharge current rapidly increases and reaches a maximum value (about $10 \mathrm{~A}$ ) at 20-25 $\mu$ s. Then it slowly

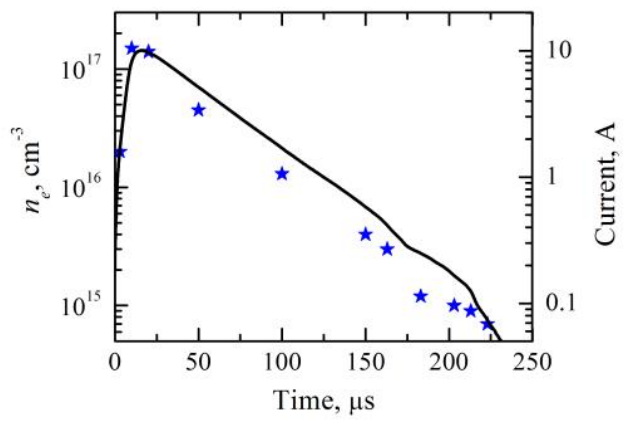

Fig. 2. Time evolutions of electron concentration (stars) and discharge current.

decreases and disappears after $250 \mu$ s.

Electron concentration in plasma columns is determined using the $\mathrm{H}_{\alpha}$ line shapes registered at different discharge time. The hydrogen $\mathrm{H}_{\alpha}$ line broadening is maximal $(>1 \mathrm{~nm})$ around $20 \mu$ s at the discharge current maximum. Estimations of the $\mathrm{H} \alpha$ line half widths, which are caused by different broadening mechanisms (instrumental, Doppler and Van-der-Waals profiles) are shown to be less than experimental profile by one-two orders of magnitude. Therefore, the main broadening of the $\mathrm{H}_{\alpha}$ line is due to the Stark broadening. According to [5], a half width $\Delta \lambda$ of $\mathrm{H}_{\alpha}$ line, broadened due to the electrical field of ions, depends on the plasma electron density $\mathrm{n}_{\mathrm{e}}\left(\mathrm{cm}^{-3}\right)$ as follows:

$$
\Delta \lambda=2.5 \cdot 10^{-9} \cdot \alpha_{H \alpha} \cdot n_{e}^{2 / 3},
$$

where $\alpha_{\mathrm{H} \alpha}$ is the Stark broadening parameter. The result is shown in Fig. 2 (stars). It is seen that the electron concentration reaches its maximum $\left(>10^{17} \mathrm{~cm}^{-3}\right)$ at the $20^{\text {th }} \mu \mathrm{s}$, corresponding to the discharge current maximum, and then slowly decreases to less than $10^{15} \mathrm{~cm}^{-3}$ at $200 \mu$ s proportionaly to the current discharge

In order to obtain the transmission spectrum, we used the spectrum analyzer of pulse signals $\mathrm{S} 4-80$ at $3 \mu \mathrm{s}$ gating. Measurements were taken step-by-step at different frequencies at the discharge current maximum (20 $\mu$ s after ignition) and at the end of the discharge (200 $\mu \mathrm{s})$, see Fig. 3. Transmission spectrum of $1 \mathrm{D}$ EBG structure formed by three inhomogeneities in $\mathrm{X}$ - waveguide is discussed in detail in [3]. It consists of the alternating pass- and stopbands. At the $20^{\text {th }} \mu \mathrm{s}$ the transmission spectrum is well contrasting. Thus in the bandpass (around $11.5 \mathrm{GHz}$ ) the attenuation of the MW signal is only about $4 \mathrm{~dB}$, while it is reduced by more

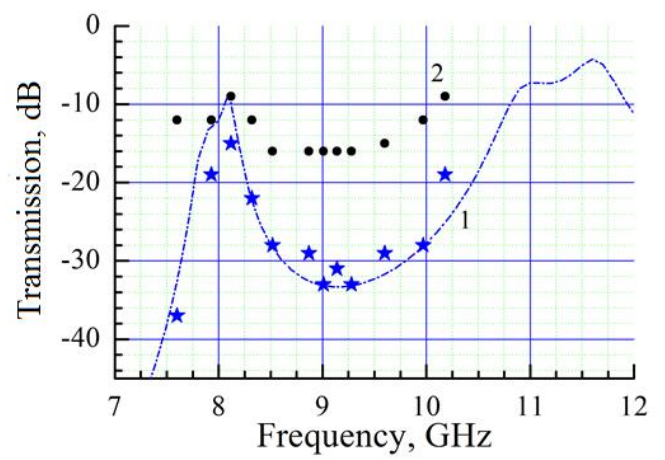

Fig. 3. Transmission spectra (experiment: stars $-20 \mu \mathrm{s}$, circles $-200 \mu \mathrm{s}$; simulation by HFSS [5]: dashed electron concentration is $10^{16} \mathrm{~cm}^{-3}$, effective diameters $1.4 \mathrm{~mm})$.

than $30 \mathrm{~dB}$ in the stopband (around $9 \mathrm{GHz}$ ). There is a second bandpass at frequency of about $8 \mathrm{GHz}$, however, attenuation of the MW signal is significant (about -10 $\mathrm{dB})$. At longer times, at $200 \mu \mathrm{s}$, the transmission signal attenuation is less expressed since the suppression of the MW signal in the stopband is only $-20 \mathrm{~dB}$. At the same time, MW signal in bandpass (around $11.5 \mathrm{GHz}$ ) decreases significantly less, only about $1 \mathrm{~dB}$.

The 1D EBG structure including pulsed plasma discharges in tubes can be irradiated by two different MW signals at frequency $9.15 \mathrm{GHz}$ : powerful pulses (about $50 \mathrm{~kW}$, pulse duration of about $150 \mathrm{~ns}$ and repetition frequency of $2 \mathrm{kHz}$ ) and a continuous microwave $(\mathrm{CW})$ signal at low power $(5 \mathrm{~mW})$. The transmited MW power normalized by the incident power is shown in Fig. 4 at low CW power (solid curve). It is seen that the transmitted signal is reduced by 3 orders of magnitude at the $25^{\text {th }} \mu \mathrm{s}$. After the $150^{\text {th }} \mu \mathrm{s}$ the transmission begins to recover and fully recovered after $230^{\text {th }} \mu$ s. Therefore, the duration of transmitted signal suppression correlates with time of plasma existing, when its concentration exceeds $10^{15} \mathrm{~cm}^{-3}$ (Fig. 2).

After that, the transmittance behaviour at high MW power was investigated. The repetition frequency of high power pulses is constant and equals $2 \mathrm{kHz}$. At the same time, the discharge repetition frequency could be only a few Hz. We had not the opportunity to synchronize these two devices at this stage. Therefore, the following scheme for recording the transmitted MW signal was chosen. Pulse voltage source and the oscilloscope were

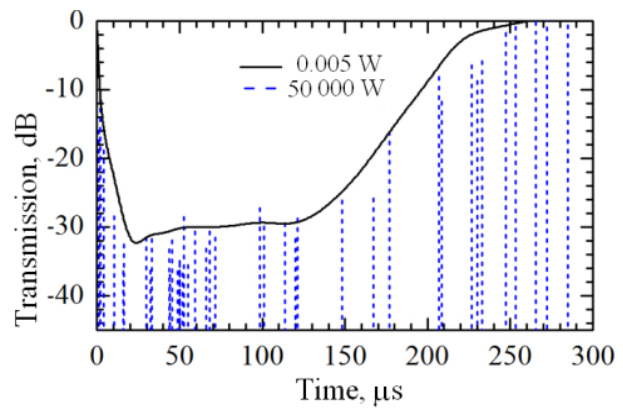

Fig. 4. Time dependence of $1 \mathrm{D}$ EBG plasma structure transmission at low (solid) and high (vertical dached lines) MW power. 
started synchronously, and a oscillogram was recorded for given time interval. An envelope of short MW pulse is appeared randomly in all of these oscillograms. Having received a set of such oscillograms and combining them, we obtained a series of random pulses. Pulses amplitudes are corresponding to the transmittance in the moment of the pulse passing through a EBG plasma structure. The results of this procedure are shown in Fig. 4 (vertical strokes).

Therefore, the MW signal suppression at the level of $-30 \mathrm{~dB}$ in forbidden band is demonstrated for low MW power of $5 \mathrm{~mW}$ and for high power of $50 \mathrm{~kW}$. The transmission spectrum switching rate is evaluated as a function of the discharge parameters (type of gas, flow rate, applied pulse voltage). A switching time of 10-20 ns and a repetition frequency up to $20 \mathrm{kHz}$ can be received.

\section{Control by 2D EBG structure}

\subsection{Experimental arrangement}

A triangle 2D EBG structure is formed by copper rods of $140 \mathrm{~mm}$ in length and $5 \mathrm{~mm}$ in diameter which are located in parallel to each other in square lattice points (Fig. 5). The lattice spacing is $22 \mathrm{~mm}$. This arrangement allows a diagonal propagation of wave mode (around $\pm 45^{\circ}$ ) for the frequency of $9.15 \mathrm{GHz}$ when a defect is present in the front row, while it is forbidden for the principal propagating mode ( $0^{\circ}$ direction) [2]. In order to excite the diagonal mode, it is necessary to put a defect in the front rod row of the EBG structure by removing one or two rods (Fig. 5, inset II) or by adding a rod (or plasma column) (Fig. 5, inset III).

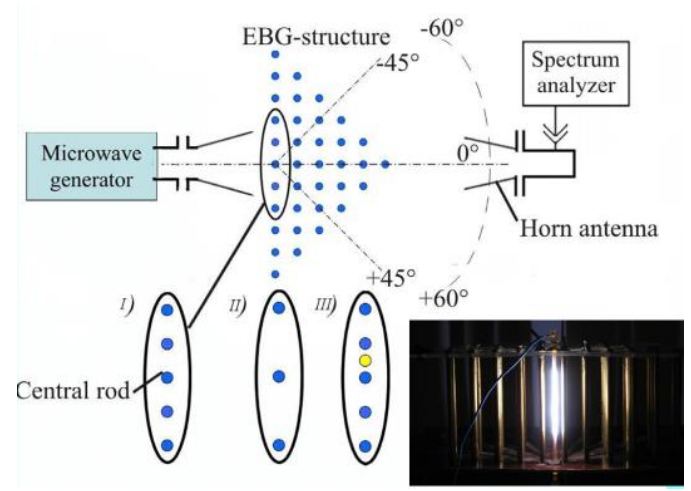

Fig. 5. Schematic of the experimental setup (I - perfect EBG structure, II - structure with tow vacations, III structure with additional defect). The inset (bottom-right) - photo of extended discharge in 2D EBG metallic

The structure is irradiated by continuous MW radiation at frequencies in the range of 8.5-10.0 GHz and power of $5 \mathrm{~mW}$ using a horn antenna. MW radiation transmitted through the 2D EBG structure is received by another horn antenna. Receiving antenna with a waveguide-to-radio adapter is moved along a circular arc of $0.5 \mathrm{~m}$ radius, centered in the middle of the triangular periodic structure base. This movement is realized within an angle range of $\pm 60^{\circ}$ relative to the axis of the radiating horn antenna (Fig. 5).

\subsection{Results and discussion}

In this study the directional pattern of this $2 \mathrm{D}$ EBG structure at frequency of $9.15 \mathrm{GHz}$ using a low-power generator $(5 \mathrm{~mW})$ operating in continuous regime was investigated. When the structure has no defect (Fig. 6, solid), there is only weak MW radiation reaching the receiving antenna, where the signal level is less by 3-4 orders of magnitude than the MW radiation signal from

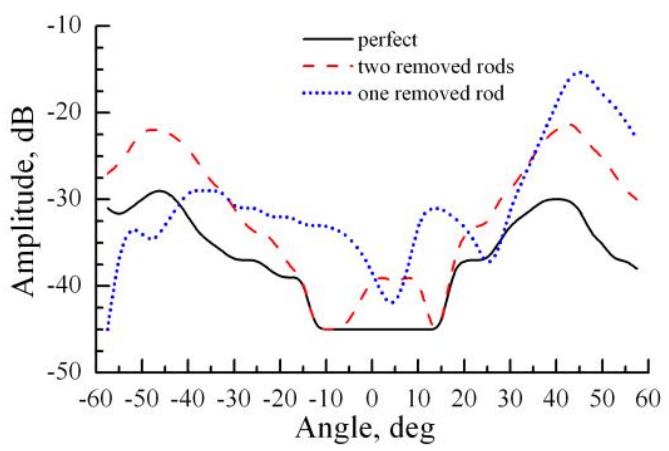

Fig. 6. Directional patterns of the 2D EBG structure.

the radiating horn antenna without $2 \mathrm{D}$ EBG structure. If the two nearest rods to the central rod in the first row are removed, MW radiation begins to propagate in directions $\pm 45^{\circ}$ and signal level grows up by $7 \mathrm{~dB}$ (dashed curve). If there is only one rod removed, a MW signal increases by $15 \mathrm{~dB}$ (dotted) at the removed rod side. The same behavior takes place in directional diagram in case of additional defect as well.

After that the plasma columns were placed as additional defects. A photo of a discharge placed in a two-dimensional electromagnetic crystal as an additional defect is shown in Fig. 5 (insert right below). Since the aperture of the irradiating antenna is $110 \times 50 \mathrm{~mm}$, the discharge gap is $50 \mathrm{~mm}$. The inner diameter of the tube is $2.5 \mathrm{~mm}$, the diameter of the electrodes is $2 \mathrm{~mm}$. The discharge is powered by a switching power supply, which is shown schematically in Fig. 1 for discharges in a one-dimensional structure.

An estimation of the electron concentration in a pulsed discharge was also performed by the Stark broadening of the hydrogen line contour $\mathrm{H} \alpha$, which was recorded experimentally with a time scan. The electron concentration was calculated according to formula (2) and its time dependence in a plasma of an extended pulsed discharge in argon at atmospheric pressure is demonstrated in Fig. 7. It is shown that the electron concentration maximum is at $20^{\text {th }} \mu \mathrm{s}$, and the maximum concentration is about $7 \times 10^{16} \mathrm{~cm}^{-3}$. After that the concentration decreases exponentially to $6 \times 10^{15} \mathrm{~cm}^{-3}$ at about $400^{\text {th }} \mu \mathrm{s}$. 
When the crystal is irradiated with $\mathrm{CW}$ radiation of about $5 \mathrm{~mW}$ power, transmission of crystal in the direction of $45^{\circ}$ changes during the first $300 \mu$ s when electron density in tubes is higher than $10^{16} \mathrm{~cm}^{-3}$. This is

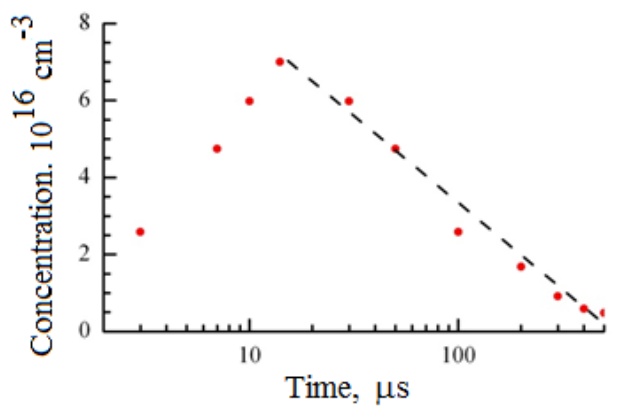

Fig. 7. Time dependence of the electron concentration in a plasma of an extended pulsed discharge $(5 \mathrm{~cm}$ long and 2.5 mmdiameter) in argon at atmospheric pressure

the case in both configurations, when the plasma column is in place of the additional inhomogeneity (curve 2 in Fig. 8) or when the plasma is in vacancy place (curve 2 in Fig. 9). After $300 \mu$ s, the electron concentration in the discharge channel is less than $10^{16} \mathrm{~cm}^{-3}$ (Fig. 6), indeed the plasma has no more effect on EBG structure.

Now, we investigate the case where the structure is irradiated by high power (about $50 \mathrm{~kW}$ ) MW pulses at $9.15 \mathrm{GHz}$ frequency and about $150 \mathrm{~ns}$ duration. As in case of $1 \mathrm{D}$ EBG structure, a waveform registration is synchronized with discharge ignition, and envelopes of MW pulses are randomly distributed during every registration time interval. Summarizing these waveforms we obtained a set of pulses distributed frequently in time scale (Fig. 8, vertical dashed lines). It is shown that the transmission change of an electromagnetic crystal for both types of defects is maximal in the discharge phase, when the electron concentration is higher than $10^{16} \mathrm{~cm}^{-3}$, and is leveled to $400 \mu \mathrm{s}$, which is later than at low MW power. Probably, it is connected with the maintenance of

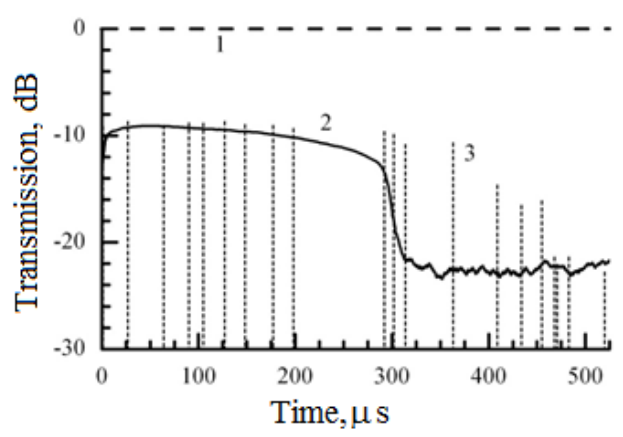

Fig. 8. The time dependence of the transmission at a $9.15 \mathrm{GHz}$ frequency of a two-dimensional electromagnetic crystal in the direction of $45^{\circ}$ with an extended pulse discharge as an additional defect. 1 - without EBG, 2 $5 \mathrm{~mW}$ and $3-50 \mathrm{~kW}$ pulses.

the electron concentration in the plasma inhomogeneity due to the collision absorption in the plasma of the transmitted powerful MW radiation. It should be noted that the maximum change $(14 \pm 2 \mathrm{~dB})$ of the transmitted MW signal in the $45^{\circ}$ direction from the defect side is observed approximately from $5 \mu$ s to $100 \mu$ s relative to the leading edge of the discharge current pulse, and it is the same as in the case of metallic inhomogeneities use.

\section{Conclusions}

The control possibilities of powerful MW by the $1 \mathrm{D}$ and 2D EBG plasma structures were demonstrated experimentally. 1D EBG plasma structure in the waveguide formed solely by pulse discharges at atmospheric pressure allows to obtain a MW signal suppression up to $40 \mathrm{~dB}$ in forbidden band and its decrease by about $4 \mathrm{~dB}$ in passband by variation of electron density in the range of $10^{14}-10^{16} \mathrm{~cm}^{-3}$. $1 \mathrm{D}$ EBG plasma structure can be used as quickly switched (10$20 \mathrm{~ns}$ ) attenuator, modulator, frequency switcher, filter and etc. for high power microwaves.

Also we demonstrated one plasma column as a key element, allowing to change the transmitted properties of regular metallic structure using 2D EBG structure.

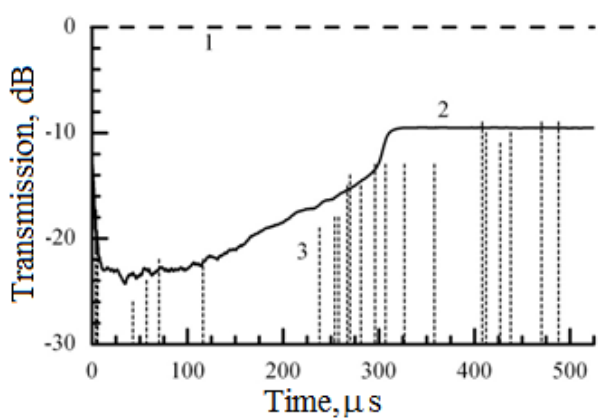

Fig. 9. The time dependence of the transmission at a $9.15 \mathrm{GHz}$ frequency of a two-dimensional electromagnetic crystal in the direction of $45^{\circ}$ with an extended pulse discharge as a defect compensator. 1 - without EBG, 2 $5 \mathrm{~mW}$ and $3-50 \mathrm{~kW}$ pulses.

When the discharge is used as an additional defect, the radiation is transmitted through the structure in $(45.0 \pm 2.5)^{\circ}$ direction. On the contrary, transmission disappears in that direction when the discharge is used as a defect compensator. The transmission changes were about $15 \mathrm{~dB}$ in both cases.

The work was partially supported by the grants the number F15F-004 from BRFFR and PICS number 151539 from CNRS.

\section{References}

1. O. Sakai, K. Tachibana, Plasma Sources Sci. Techn., 21(1) (2012)

2. J. Lo, J.Sokoloff et al., Applied Physics Letters, 96 (2010)

3. V.I. Arkhipenko et al., Journal of applied physics, 116(12) (2014)

4. H.R. Griem, "Spectral lines broadening" (Academic Press, New York and London, 1974).

5. Ansoft HFSS, http://www.ansys.com/Products/ Simulation+Technology/Electromagnetics/HighPerformance+Electronic+Design/ANSYS+HFSS 